\title{
An Atomistic Look into Bio-inspired Nanoparticles and their Molecular Interactions with Cells
}

\author{
Emanuele Petretto ${ }^{a}$, Pablo Campomanes ${ }^{a}$, Francesco Stellaccib, Barbara Rothen-Rutishauser ${ }^{c}$, Alke \\ Petri-Fink ${ }^{\mathrm{cd}}$ and Stefano Vanni ${ }^{\star a}$
}

\begin{abstract}
Nanoparticles (NPs) have sizes that approach those of pathogens and they can interact with the membranes of eukaryotic cells in an analogous fashion. Typically, NPs are taken up by the cell via the plasma membrane by receptor-mediated processes and subsequently interact with various endomembranes. Unlike pathogens, however, NPs lack the remarkable specificity gained during the evolutionary process and their design and optimization remains an expensive and time-consuming undertaking, especially considering the limited information available on their molecular interactions with cells. In this context, molecular dynamics (MD) simulations have emerged as a promising strategy to investigate the mechanistic details of the interaction of NPs with mammalian or viral membranes. In particular, MD simulations have been extensively used to study the uptake process of NPs into the cell, focusing on membrane vesiculation, endocytic routes, or passive permeation processes. While such work is certainly relevant for understanding NP-cell interactions, it remains very difficult to determine the correspondence between generic models and the actual NP. Here, we review how chemicallyspecific MD simulations can provide rational guidelines towards further bio-inspired NP optimization.
\end{abstract}

Keywords: Cellular membranes · Molecular dynamics · Nanoparticle

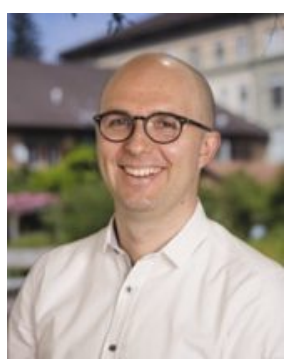

Stefano Vanni studied Physics at the University of Bologna, Italy, where he received his BSc and MSc in Theoretical Physics. Later, he obtained his PhD in the field of computational chemistry and biochemistry at EPF, Lausanne, before joining the Institut de Pharmacologie Cellulaire et Moleculaire at CNRS in Sophia Antipolis, France, as a postdoctoral fellow. Since 2015, he is a tenured researcher at CNRS, and since 2017, he is a Swiss National Science Foundation Associate Professor in the Department of Biology at the University of Fribourg. Since 2018 he is affiliated to the NCCR in Bio-Inspired Materials. His main research focus is the molecular understanding of the role of membrane and lipid physicochemical properties in intracellular trafficking processes and lipid metabolism.

\section{Introduction}

Nanoparticles (NPs) have emerged as a powerful class of materials due to the plethora of their potential applications. Because of their nanometer scales, NPs are characterized by an extremely high surface-to-volume ratio and, as a direct consequence, sizedependent properties that are not observed for mesoscale objects become relevant for NPs (Fig. 1). Thus, surface chemistry and interfacial properties dominate the interaction of NPs with the surrounding environment. ${ }^{[1,2]}$

Among NPs, metal NPs have been thoroughly studied for their simple synthesis, tunable size, and prominent optical features such as surface plasmon resonance. ${ }^{[3]}$ Bare NPs can be surface

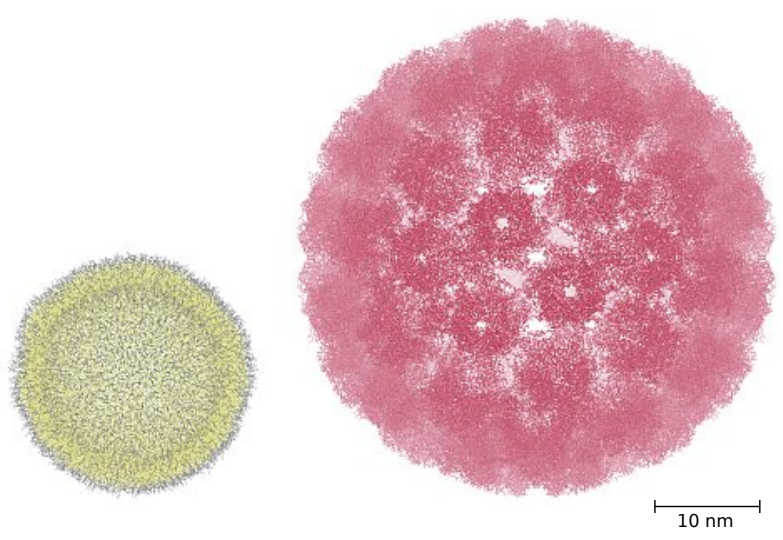

Fig. 1. Comparative dimensional representation of a monolayer passivated NP (left), a small DOPC vesicle (center), and of human papillomavirus 16 capsid (right).

functionalized $e . g$. to enhance colloidal stability or confer functionality to the NPs using standard bioconjugation approaches. ${ }^{[4]}$

Nowadays, NPs have different technological applications and can be used in the biomedical field for biosensing, imaging, targeted interaction, and theranostic purposes. Since these NP-cell interactions depend heavily on the NP type, size, surface-chemistry etc., an in-depth knowledge of the particles and their surface properties is desirable to both allow for a safe implementation and tune their biological interactions.

In this context, Molecular Dynamics (MD) simulations have emerged as a promising computational approach to investigate 
the physicochemical properties of NPs, and especially their interactions with biological structures such as proteins and cellular membranes. ${ }^{[5]}$ In fact, MD simulations are capable of accurately describing molecular systems by (i) taking into account the kinetic and potential energy of the individual atoms that constitute the molecules under investigation and (ii) by studying their dynamical properties under the assumption that these molecules follow Newton's equations of motion. ${ }^{[6]}$

The molecular models used to describe NPs with MD simulations can be classified into two main categories: all-atom (AA) models and coarse grain (CG) models. ${ }^{[6]}$ AA models use an explicit representation of all atoms, with the interactions between them fitted on either from high-level quantum-mechanical calculations or known experimental data. This methodology allows for the accurate characterization of molecular processes involving, for example, non-bonded intermolecular interactions or conformational changes, but suffers from performance limitations in both time and space dimensions when dealing with large systems due to intrinsically high computational demands.

To overcome these limitations, chemical-specific coarsegrained (CG) models have been developed to investigate molecular systems approaching size scales of the order of hundreds of nanometers. ${ }^{[7]}$ In CG models, the description of molecules is simplified by grouping multiple atoms in pseudo-atoms, named beads. CG force field parameters are commonly determined by fitting to results obtained from AA simulations or via direct comparison with experimental properties. While this procedure leads to a lower spatial resolution, it allows to investigate the structure and dynamics of large macromolecular systems for biologically relevant simulation times. ${ }^{[6]}$

In the last few years, both AA and CG MD simulations have provided major advances in our understanding of NPs. ${ }^{[8-14]}$ For example, AA simulations have been able to elucidate how the presence of distinct ligands grafted on the NP surface modifies the physicochemical properties of the NP interface, including its interaction with water, ${ }^{[15]}$ or how tuning NP grafting can modulate nanoparticle-based chemosensing. ${ }^{[14]}$ In parallel, CG simulations have been successful in describing the complex interplay between NP and lipid bilayers, reproducing reasonably well the structural features of the initial contact established between the NP and the external leaflet of the membrane, as well as the subsequent NP translocation both in the absence and in the presence of cholesterol.[11,13]

In this short review, we will focus on how the use of MD simulations can increase our understanding into two interesting aspects of NPs mode of action at the interface between cellular biology and material science: how NPs interact with cellular membranes and how NPs can kill viruses by deforming their lipid envelope.

\section{Modeling Nanoparticle-Membrane Interactions}

Eukaryotic cells are characterized by a rich variety of membranes, that differ by morphology as well as by protein and lipid composition. These membranes help to compartmentalize cellular regions, and they essentially constitute the boundaries of the cell. Because of that, biological membranes selectively regulate the passage of substances in and out of the cell.[16]

In the last few years, a consensus is emerging that membranes also constitute a biochemical platform for cellular signaling, with membrane-interacting proteins as well as pathogens taking advantage of the intrinsic differences between cellular membranes. ${ }^{[17]}$ Notably, NPs have also been shown to interact extensively with membranes and they can be taken up by cells using similar pathways to those of pathogens. ${ }^{[24]}$

In this context, MD has emerged as one of the key methodologies to better characterize the interactions between cellular membranes and NPs, since this approach offers the possibility to investigate the behavior of matter directly at the atomistic level under highly controlled conditions. ${ }^{[5]}$ In particular, molecular simulations have been extensively used to clarify which mechanisms drive the uptake process of NPs into the cell, focusing on membrane vesiculation, endocytic routes, or - what also has been described for certain NPs - passive permeation processes. ${ }^{[5]}$ While such work is certainly relevant for understanding NP-cell interactions, it remains very difficult to determine the correspondence between generic models and the actual nanomaterials prepared in experimental laboratories. To this end, more accurate chemical-specific MD simulations have been used in the last few years to take fully into account the influence of NP surface chemistry (Fig. 2). ${ }^{[8-14]}$

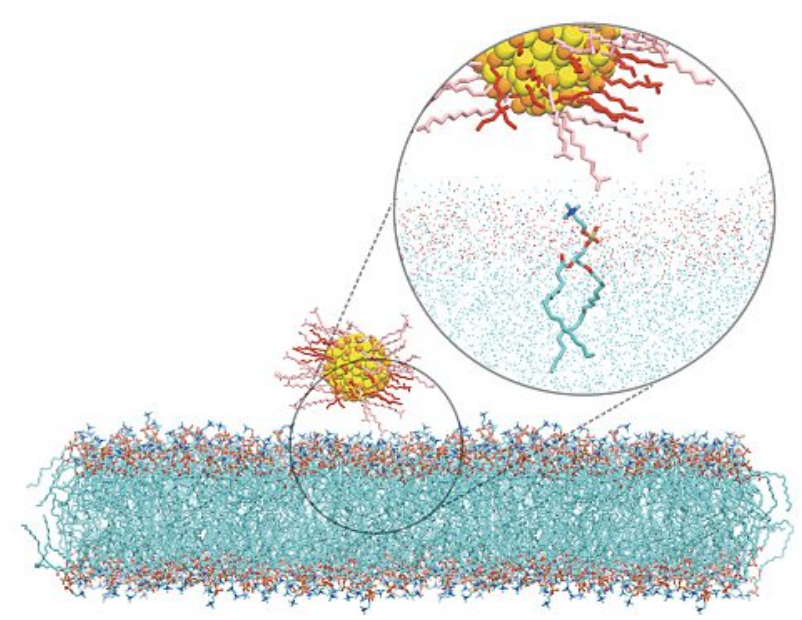

Fig. 2. Representative snapshot of a striped monolayer passivated AuNP in water approaching a lipid bilayer. NP: pink, mercapto undecane carboxylate; red, octanethiol; orange, sulfur; yellow, gold. Lipids: blue, nitrogen; brown, phosphorus; red, oxygen; cyan, carbon.

In this context, MD simulations have been paramount to show that the interaction between NPs and membranes can occur either via adhesion, penetration, or wrapping and that different structural NP properties influence its permeation process. For example, in agreement with experimental studies, ${ }^{[21]}$ simulations have shown that translocation is favored for smaller NPs, since the minimum driving pressure required for this process increases for increasing NP sizes. ${ }^{[18]}$ Shape also might play a significant role in permeation, and MD simulations have been used to determine the minimal driving force of penetration of different shaped NPs. ${ }^{[19,22]}$ Anisotropic NPs, for example, have been shown to possess the minimum driving force, ${ }^{[18,19]}$ since their penetration generates a high local curvature that varies along the translocation process. The grafting pattern (striped, random, and homogenous ligands) also greatly influence translocation, as it does the hydrophobic length of the alkyl-functionalization, with longer ligands promoting penetration and wrapping phases, while simultaneously reducing the complete translocation due to the affinity with the hydrophobic tail of lipids.

While these approaches still have major limitations, for example failing to correctly reproduce the translocation mechanism of NPs across model membranes due to inconsistencies in reproducing free energy barriers, ${ }^{[8]}$ they have shown the potential of $\mathrm{MD}$ simulations to investigate NP-membrane interactions, and they can be potentially reproduced in more biologically relevant contexts. In the endocytic pathway, for example, NP internalization occurs via the invagination and the inward budding of a small region of the membrane. It is acknowledged that endocytosis is the principal pathway used by cells for the NPs internalization, [24] but this has major drawbacks for biomedical applications since endocytosed NPs are rapidly transported into lysosomes. This organelle is responsible for the breakdown and degradation of many molecules and the fate of NPs after their lysosomal entry, as well as its dependence on the molecular properties of NPs, needs to 
be further characterized. There, MD simulations can potentially help understand the influence of particle size and passivation in the mechanism of endo-lysosomal entry. In more general terms, in silico simulations can provide an invaluable support to the experimental investigations by providing a detailed atomistic or molecular interpretation of the mechanism of translocation of NPs inside or across the membrane, hopefully to develop NPs with high internalization capacity and low cytotoxicity.

\section{Modeling NP-induced Virucidal Activity}

Gold NPs have emerged as a very promising candidate for diagnostic and therapeutic applications for two main reasons: (i) their low cytotoxicity and high biocompatibility and (ii) the large number of surface chemistries that they can easily be functionalized with. Recently, a class of gold NPs has been shown to have potent virucidal activity, i.e. they cause irreversible viral deactivation, with in vitro nanomolar activity against several viruses, including HSV, PV, RSV, Dengue, Lenti, and Zika, and ex vivo activity in human cervicovaginal histocultures infected by HSV-2. ${ }^{[23]}$

As is the case for several substances that are planned to achieve broad-spectrum efficacy, the basic idea behind the development of these NPs was to target virus-cell interactions that are common to many viruses. ${ }^{[25,26]}$ In particular, these NPs mimic the interaction between the viral attachment ligand and its associated cell receptor responsible for the first step of the virus replication cycle, in this case heparan sulfate proteoglycans (HSPGs). Since HSPGs are expressed on the surface of almost all eukaryotic cell types, it is not surprising that many viruses, including HIV-1, HSV, HCMV, HPV, RSV, and Flavivirus exploit HSPGs as the target of their attachment ligand. [23]

The binding between viruses and HSPGs occurs via the interaction of closely-packed arrangements of multiple basic amino acids on the proteins, which constitute the viral attachment ligand, with the negatively charged sulfated groups of heparan sulfate (HS) in the glycocalix of the cell surface. Thus, since most viral attachment ligands have binding domains composed of close packed repeating units, the basic idea behind the NPs development was to engineer its linkers in order to promote multivalent binding between the NPs and the viral attachment ligands. To do so, the NPs are coated with long linkers composed of undecanesulfonic acid (MUS), to achieve strong irreversible multivalent binding.

Upon binding, this family of gold NPs leads to local distortions and eventually to a global virus deformation, with the consequent irreversible loss of infectivity. ${ }^{[23]}$ Remarkably, these NPs show in vitro inhibition of many HSPG dependent viruses either enveloped (HSV, RSV, Lentivirus, Dengue, and Zika virus) or naked (HPV). While it is known that NPs can lead to significant local distortions when binding strongly to a membrane, the underlying mechanism of membrane deformation remains unclear in the specific case of a viral capsid.

In this context, MD simulations should help clarify the mechanism leading to membrane deformation and rupture. In detail, several aspects of this process can be thoroughly investigated using particle-based modelling approaches, and namely (i) how the ligands present on the surface of the NP directly interact with the lipids of the viral envelope, (ii) whether and how NPs aggregate within the hydrophobic core of the membrane, (iii) whether membrane deformation can be promoted by NP crowding, and (iv) whether membrane deformation might originate from forcepulling by NP-receptors interactions. Ideally, by performing MD simulations to explicitly investigate the mechanism of interaction between MUS-coated gold NPs and a model membrane mimicking the viral envelope, the relevance and energetic profile of these mechanisms should be properly quantified, potentially leading to the tuning of NPs properties along several directions, including (i) the replacement of gold by a more biocompatible core and (ii) the optimization of the length of the sulfonate linkers and NPs size.

\section{Summary}

In this short report, we focused on how MD simulations might help elucidate key cellular processes that involve NP-membrane interactions. Experimental studies suggest that the physicochemical properties of NPs, including size, hydrophilic-hydrophobic coating characteristics, and topological organization of ligands on the nanoparticle surface are crucial to tune NPs interaction with lipid membranes. Because of their intrinsically high resolution, MD simulations are extremely well adapted to investigate these properties with molecular detail. We expect that these methodologies will be paramount to elucidate several mechanistic aspects of $\mathrm{NP}$-cell interactions that still escape our complete understanding.

\section{Acknowledgements}

SV, EP, BRR and APF acknowledge support by the Swiss National Science Foundation through the National Center of Competence in Research Bio-Inspired Materials, PC acknowledges support from the Novartis Foundation for Biomedical Research grant \#17C139.

\section{Received: November 15, 2018}

[1] A. Verma, F. Stellacci, Small 2010, 6, 12 .

[2] D. Septiadi, F. Crippa, T. L. Moore, B. Rothen-Rutishauser, A. Petri-Fink, Adv. Mater. 2018, 30, 1704463.

[3] L. C. Kennedy, L. R. Bickford, N. A. Lewinski, A. J. Coughlin, Y. Hu, E. S. Day, J. L. West, R. A. Drezek, Small 2011, 7, 169.

[4] E. C. Dreaden, A. M. Alkilany, X. Huang, C. J. Murphy, M. A. El-Sayed, Chem. Soc. Rev. 2012, 41, 2740.

[5] G. Rossi, L. Monticelli, Biochim. Biophys. Acta-Biomembr. 2016, 1858.

[6] M. Cascella, S. Vanni, Chem. Model. 2015, 12, 1.

[7] T. A. Soares, S. Vanni, G. Milano, M. Cascella, J. Phys. Chem. Lett. 2017, 8, 3586.

[8] S. Salassi, F. Simonelli, D. Bochicchio, R. Ferrando, G. Rossi, J. Phys. Chem. C 2017, 121, 10927

[9] F. Simonelli, D. Bochicchio, R. Ferrando, G. Rossi J. Phys. Chem. Lett., 2015, 6, 16.

[10] R. C. Van Lehn, A. Alexander-Katz, J. Phys. Chem. C 2013, 117, 20104.

[11] R. C. Van Lehn, P. U. Atukorale, R. P. Carney, Y. S. Yang, F. Stellacci, D. J. Irvine, A. Alexander-Katz, Nano Lett. 2013, 13, 4060.

[12] Q. Ong, Z. Luo, F. Stellacci, Acc. Chem. Res. 2017, 50, 1911.

[13] P. Angelikopoulos, L. Sarkisov, Z. Cournia, P. Gkeka, Nanoscale 2017, 9 , 1040.

[14] L. Riccardi, L. Gabrielli, X. Sun, F. De Biasi, F. Rastrelli, F. Mancin, M. De Vivo, Chem. 2017, 3, 92.

[15] J. J. Kuna, K. Voitchovsky, C. Singh, H. Jiang, S. Mwenifumbo, P. K. Ghorai, M. M. Stevens, S. C. Glotzer, F. Stellacci, Nat. Mater. 2009, 8, 837.

[16] G. van Meer, D. R. Voelker, G. W. Feigenson, Nat. Rev. Mol. Cell Biol. 2008, 9, 112 .

[17] R. Friedman, S. Khalid, C. Aponte-Santamaría, E. Arutyunova, M. Becker, K. J. Boyd, M. Christensen, J. T. S. Coimbra, S. Concilio, C. Daday, F. J. van Eerden, P. A. Fernandes, F. Gräter, D. Hakobyan, A. Heuer, K. Karathanou, F. Keller, M. J. Lemieux, S. J. Marrink, E. R. May, A. Mazumdar, R. Naftalin, M. Pickholz, S. Piotto, P. Pohl, P. Quinn, M. J. Ramos, B. Schiøtt, D. Sengupta, L. Sessa, S. Vanni, T. Zeppelin, V. Zoni, A.-N. Bondar, C. Domene, J. Membr. Biol. 2018, 251, 609.

[18] K. Yang, Y.-Q. Ma, Nat. Nanotechnol. 2010, 5, 579.

[19] S. Dasgupta, T. Auth, G. Gompper, Nano Lett. 2014, 14, 687.

[20] H. Ding, Y. Ma, Sci. Rep. 2016, 6, 26783.

[21] Y. Jiang, S. Huo, T. Mizuhara, R. Das, Y.-W. Lee, S. Hou, D. F. Moyano, B. Duncan, X.-J. Liang, V. M. Rotello, ACS Nano 2015, 9, 9986.

[22] A. R. Tao, S. Habas, P. Yang, Small 2008, 4, 310.

[23] V. Cagno, P. Andreozzi, M. D’Alicarnasso, P. Jacob Silva, M. Mueller, M. Galloux, R. Le Goffic, S. T. Jones, M. Vallino, J. Hodek, J. Weber, S. Sen, E.-R. Janeček, A. Bekdemir, B. Sanavio, C. Martinelli, M. Donalisio, M.-A. Rameix Welti, J.-F. Eleouet, Y. Han, L. Kaiser, L. Vukovic, C. Tapparel, P. Král, S. Krol, D. Lembo, F. Stellacci, Nat. Mater. 2017, 17, 195.

[24] N. Oh, J. H. Park, Int. J. Nanomedicine 2014, 9 Suppl 1, 51.

[25] D. Baram-Pinto, S. Shukla, N. Perkas, A. Gedanken, R. Sarid, Bioconjug. Chem. 2009, 20, 1497.

[26] M. Li, L. Gao, C. Schlaich, J. Zhang, I. S. Donskyi, G. Yu, W. Li, Z. Tu, J. Rolff, T. Schwerdtle, R. Haag, N. Ma, ACS Appl. Mater. Interfaces 2017, 9 , 35411 . 